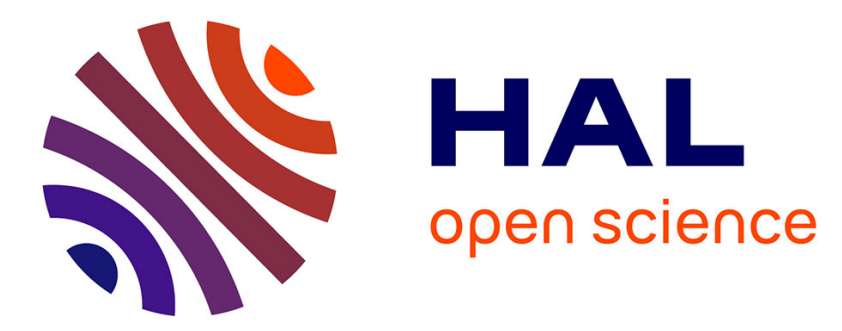

\title{
Detection of Highly Ionized C IV Gas Within the Local Cavity
}

\author{
Barry Y. Welsh, Jonathan Wheatley, Oswald H. W. Siegmund, Rosine \\ Lallement
}

\section{- To cite this version:}

Barry Y. Welsh, Jonathan Wheatley, Oswald H. W. Siegmund, Rosine Lallement. Detection of Highly Ionized C IV Gas Within the Local Cavity. The Astrophysical journal letters, 2010, 712 (2), pp.L199L202. 10.1088/2041-8205/712/2/L199 . hal-00477802

\section{HAL Id: hal-00477802 https://hal.science/hal-00477802}

Submitted on 14 Nov 2019

HAL is a multi-disciplinary open access archive for the deposit and dissemination of scientific research documents, whether they are published or not. The documents may come from teaching and research institutions in France or abroad, or from public or private research centers.
L'archive ouverte pluridisciplinaire HAL, est destinée au dépôt et à la diffusion de documents scientifiques de niveau recherche, publiés ou non, émanant des établissements d'enseignement et de recherche français ou étrangers, des laboratoires publics ou privés. 


\title{
DETECTION OF HIGHLY IONIZED C IV GAS WITHIN THE LOCAL CAVITY
}

\author{
Barry Y. Welsh ${ }^{1}$, Jonathan Wheatley ${ }^{1}$, Oswald H. W. Siegmund ${ }^{1}$, and Rosine Lallement ${ }^{2}$ \\ ${ }^{1}$ Space Sciences Laboratory, University of California, 7 Gauss Way, Berkeley, CA 94720, USA \\ ${ }^{2}$ IPSL/LATMOS, Versailles, France \\ Received 2010 February 8; accepted 2010 March 4; published 2010 March 17
}

\begin{abstract}
We present high resolution $(R=114,000)$ ultraviolet measurements of the interstellar absorption line profiles of the C IV $(1550 \AA)$ high ionization doublet recorded toward the nearby B2Ve star HD $158427(d \sim 74 \mathrm{pc})$. These data, which were recorded with the recently re-furbished Space Telescope Imaging Spectrograph instrument on the Hubble Space Telescope, represent the most convincing detection yet of highly ionized C IV absorption that can be associated with interstellar gas located within the boundary of the Local Cavity (LC). Two highly ionized gas clouds at $V_{1}=-24.3 \mathrm{~km} \mathrm{~s}^{-1}$ and $V_{2}=-41.3 \mathrm{~km} \mathrm{~s}^{-1}$ are revealed in both $C_{\text {IV }}$ absorption lines, with the $V_{1}$ component almost certainly being due to absorption by the Local Interstellar Cloud $(d<5 \mathrm{pc})$. Although the observed column densities for both cloud components can be explained by the predictions of current theoretical models of the local interstellar medium, the narrow Doppler width of the $V_{2}$ line profile $\left(b=6.8 \mathrm{~km} \mathrm{~s}^{-1}\right)$ indicates an unusually low gas temperature of $\leqslant 34,000 \mathrm{~K}$ for this highly ionized component. It is conjectured that the $V_{2}$ cloud may be due to an outflow of highly ionized and hot gas from the nearby Loop I superbubble. These new data also indicate that absorption due to highly ionized gas in the LC can be best described as being "patchy" in nature.
\end{abstract}

Key words: ISM: clouds

Online-only material: color figures

\section{INTRODUCTION}

The volume of the interstellar medium (ISM) that surrounds the Sun to a radius of $\sim 80 \mathrm{pc}$ in most directions, often called the Local Cavity (LC), is known to possess an unusually low neutral gas density of $n_{\mathrm{H}_{\mathrm{I}}}<0.1 \mathrm{~cm}^{-3}$. However, the physical characteristics of the plasma that fills this interstellar void are still a matter of vigorous debate. The traditional view of this rarefied interstellar region is that it is filled with a soft $\mathrm{X}$-ray emitting plasma with a temperature of $\sim 1$ million $\mathrm{K}$ (Snowden et al. 1998), with the additional presence of numerous interspersed warm $(T \sim 7000 \mathrm{~K})$ and partially ionized diffuse cloudlets (Lallement et al. 1986; Redfield \& Linsky 2008). However, over the past decade several observational inconsistencies have questioned the validity of this picture of a local hot and highly ionized interstellar bubble. For example, solar wind charge exchange reactions in the heliosphere have been shown to produce a significant fraction of the soft X-ray emission that was previously ascribed to the hot LC (Robertson \& Cravens 2003; Koutroumpa et al. 2009), and extreme ultraviolet line emission predicted by several models of a hot LC has not been detected by both the Extreme-Ultraviolet Explorer (EUVE) and CHIPS satellite missions (Jelinsky et al. 1995; Hurwitz et al. 2005). For a fuller discussion of the "hot or not" LC debate, we refer readers to Welsh \& Shelton (2009).

Strong evidence for a local highly ionized plasma with a (lower) temperature of $\sim 3 \times 10^{5} \mathrm{~K}$ has come from observations of the O VI (1032 $\AA$ ) ion, seen in the far UV absorption spectra of several stars located within 100 pc (Jenkins 1978; Savage \& Lehner 2006). This ion is generally thought to trace collisionally ionized gas that can exist in (turbulent) transition zones between the hot million K plasma of the LC and cooler gas clouds residing therein. In addition, one may also expect to detect local absorption from the high ion line doublets of Nv (1238 $\AA$ ), C IV (1550 ̊), and Si IV (1394 $\AA$ ), which can form in lower temperature gas at $\sim 10^{5} \mathrm{~K}$. Several theoretical models have been proposed to explain the observed ratios of the lines of O vi, Nv, C IV, and Si Iv for more distant regions of the ISM that invoke shocks, photoionization, evaporating interfaces, or rapidly cooling gas (Indebetouw \& Shull 2004). Typically, the population ratios of $\mathrm{N} \mathrm{v}$ and $\mathrm{C}$ IV to $\mathrm{O}$ VI lie in the range $0.1-0.4$, such that if $\mathrm{O}$ VI is well detected then the other two ions should also be present. However, there is currently only one sight line within $100 \mathrm{pc}$ (toward $\alpha$ Vir, $d=80 \mathrm{pc}$ ) against which detections of interstellar Nv, Civ, and Si IV absorption can be tested against theory (Huang et al. 1995). Although these observed column density ratios are broadly consistent with the theory of Slavin \& Cox (1992) in which cooling hot gas condenses onto the interior shell of an evolving supernova remnant bubble, we note that this (high latitude) star has a significant surrounding $\mathrm{H}_{\text {II }}$ region, as traced by its $\mathrm{H} \alpha$ and $\mathrm{S}_{\mathrm{II}}$ emission contours (Haffner et al. 2003). These high ion absorption lines are all centered near the rest-frame velocity of the star, suggesting a circumstellar rather than an interstellar origin. Therefore, other production mechanisms such as ionization of the surrounding $\mathrm{H}$ II region by hot X-ray emitting gas cannot be discounted for this sight line (Cowie et al. 1981).

One universally accepted physical characteristic of interstellar gas within the LC is the ubiquitous presence of photoionized clouds with a temperature of $\sim 10^{4} \mathrm{~K}$ (Redfield \& Linsky 2004; Lehner et al. 2003). The most well-observed of these clouds is the Local Interstellar Cloud (LIC) that surrounds most of the heliosphere (Lallement et al. 1995). The LIC is a partially ionized structure with a temperature of $\sim 6500 \mathrm{~K}$, an electron density of $0.07 \mathrm{~cm}^{-3}$ with elements such as $\mathrm{Fe}$ and $\mathrm{Mg}$ being depleted with respect to solar values (Redfield 2009). Much effort has been directed into understanding the ionization state and the potential sources and radiative transfer of ionization for the LIC (Bruhweiler \& Cheng 1988; Slavin \& Frisch 2002). The unusual ionization of the local ISM (in which $\mathrm{He}$ is more ionized than $\mathrm{H}$ ) suggests that the LIC may be out of ionization equilibrium (Lyu \& Bruhweiler 1996; Wolff et al. 1999), whereas local observa- 
tions of Ar I and $\mathrm{O}$ I indicate that the LIC is close to ionization and thermal equilibrium (Jenkins et al. 2000).

A firm prediction of many of these ionization models of the local gas is that the $\mathrm{C}$ IV ion should be formed at the evaporative interface between the LIC and the purported ambient hot plasma of the LC (Bohringer \& Hartquist 1987; Slavin \& Frisch 2002). Such models typically predict C IV column density values of $N($ C IV $)=1-3 \times 10^{12} \mathrm{~cm}^{-3}$ per interface, which translate into absorption equivalent widths which should be measurable by present UV instrumentation on telescopes such as the Hubble Space Telescope (HST). However (to our knowledge), no convincing $\mathrm{C}$ IV detection currently exists for targets whose sight lines are wholly contained within the confines of the LC. Although the apparent dearth of detectable C IV within the LC could be explained by the existence of tangential magnetic fields emanating from the walls of the LC that can quench the production sites of conduction (Cox \& Helenius 2003), it is difficult to believe that this should apply to all local clouds whose sight lines have been sampled through many UV absorption measurements toward numerous stars in the 50-150 pc range. We note that possible detections of $\mathrm{C}$ IV absorption originating within the LC have been claimed for several targets located at distances beyond the boundary of the LC. These cases include weak $\mathrm{C}$ IV absorption detected toward both $\beta \mathrm{CMa}(d=153 \mathrm{pc})$ and $\epsilon \mathrm{CMa}(d=132 \mathrm{pc})$ at an absorption velocity consistent with that of the LIC (Dupin \& Gry 1998; Gry \& Jenkins 2001). However, several authors have questioned whether this highly ionized gas arises in a more distant cloud whose velocity is similar to that of the LIC (Hebrard et al. 1999; Wood et al. 2002). In addition, C IV absorption was detected at the velocity of the G-cloud toward two B-stars $(d \sim 180 \mathrm{pc})$ in the direction of the Loop I bubble by Welsh \& Lallement (2005), but the association with very local absorption was discounted on the grounds that highly ionized gas was not detected toward two closer $(d<130 \mathrm{pc})$ stars with similar sight lines. Furthermore, we note that although the local ISM has been well sampled using hot white dwarf stars as background UV continuum sources, no detections of interstellar $\mathrm{N} v$ or $\mathrm{C}$ IV have been claimed, although detections of both photospheric and circumstellar $\mathrm{N}$ V and $\mathrm{C}$ IV currently exist (Bannister et al. 2003).

In this Letter, we report on the detection of highly ionized interstellar C IV absorption that unambiguously arises in a gas cloud located within the LC along the sight line toward the star HD $158427(d=74 \mathrm{pc})$. Based on the velocity of one of the absorption components, the highly ionized absorption can be physically associated with the LIC. This detection therefore helps constrain the possible ionization mechanisms present within the LC.

\section{OBSERVATIONS AND DATA REDUCTION}

Observations of the B2Ve star, HD 158427 ( $d=74 \pm 5 \mathrm{pc})$, were made under the NASA HST Cycle 17 Cosmic Origins Spectrograph Guaranteed Time observation program GTO11525 in 2009 October, using the recently re-furbished Space Telescope Imaging Spectrograph (STIS) instrument (Woodgate et al. 1998). The data were recorded through the $0.2 \times 0.05$ arcsec aperture (with neutral density filtering) using the E140H echelle grating. Ultraviolet photons were collected with the far UV Multi-Anode Microchannel Array (MAMA) detector during two exposures of $1760 \mathrm{~s}$ and $1838 \mathrm{~s}$ with the grating centered at $1343 \AA$ and $1598 \AA$, respectively. This resulted in spectra recorded at a resolving power of $R \sim 114,000\left(2.6 \mathrm{~km} \mathrm{~s}^{-1}\right)$ for the spectral orders observed over the entire 1245-1730 ̊ region.
The data were processed at the Multi-mission Archive of the Space Telescope Science Institute (MAST) using the CALSTIS pipeline software with the final spectra being presented in the heliocentric scale with a typical accuracy of $\sim \pm 1 \mathrm{~km} \mathrm{~s}^{-1}$.

Data for the C IV doublet $(\lambda 1548.22 \AA$ and $\lambda 1550.77 \AA)$ appear in two adjacent echelle orders, which were co-added (and weighted inversely with respect to the continuum noise) to improve the resultant signal-to-noise ratio of the data. Local continua were established for both lines and their resultant intensity absorption profiles fit simultaneously with multiple absorption components as described in Welsh \& Lallement (2005). A best fit to the doublet was obtained using a twocomponent cloud model, and in Figure 1 we show the observed residual intensity profiles for both of the C IV absorption lines together with their component fit values of column density, $N(\mathrm{C}$ IV), Doppler $b$-value and cloud component heliocentric velocity, $V$.

\section{DISCUSSION}

\subsection{Is the CIV Absorption of Interstellar Origin?}

Although we have clearly detected significant C IV absorption in the UV spectrum of HD 158427, we need to establish that it is not of a stellar origin. Firstly, we can rule out a photospheric origin for C IV based on the extreme weakness of the Si III ( $\lambda 1294 \AA$ and $\lambda 1299 \AA$ ) and C III $(\lambda 1247 \AA$ ) photospheric lines in this spectrum. Secondly, the $\mathrm{C}$ IV lines are not formed at the known radial velocity of star $\left(0 \mathrm{~km} \mathrm{~s}^{-1}\right)$, but instead one of the two absorption components (i.e., $\mathrm{V}_{1} \sim-24.3 \mathrm{~km} \mathrm{~s}^{-1}$ ) is formed within \pm $2 \mathrm{~km} \mathrm{~s}^{-1}$ of the central absorption velocities of the low ionization UV (S II, Si II, Ni II, and Fe II) and optical (Na I and Ca II) interstellar lines observed toward this star by B. Y. Welsh \& R. Lallement (2010, in preparation). We show the $\mathrm{S}_{\text {II }}(\lambda 1259 \AA)$ and Fe II $(\lambda 1608 \AA)$ absorption line profiles in Figure 1 . Thirdly, the high rotational velocity of the star $\left(V_{\text {rot }} \sim\right.$ $375 \mathrm{~km} \mathrm{~s}^{-1}$ ) also precludes a stellar origin for the observed narrow C IV absorption lines. Therefore, we believe that at least one of the observed C IV absorption components, $V_{1}$, is interstellar.

\subsection{Does the CIV Absorption Arise Within the LC?}

In Figure 2, we show the position of HD 158427 with respect to the spatial distribution of neutral gas (as traced by Na I absorption) within $100 \mathrm{pc}$ of the Sun (Welsh et al. 2010). The star is clearly well placed within the dense surrounding neutral boundary of the LC. The velocity vectors of any absorption arising in the LIC and G-clouds (Lallement et al. 1995), as projected toward HD 158427 , are $-23.1 \mathrm{~km} \mathrm{~s}^{-1}$ and $-26.5 \mathrm{~km} \mathrm{~s}^{-1}$, respectively. Thus, component $V_{1}$ can confidently be assigned to absorption arising within the LIC complex. Additionally, the column density of this component, $\log N(\mathrm{C}$ IV $)=$ $12.20 \mathrm{~cm}^{-2}$ is very similar to that assigned to LIC absorption in the sight lines toward both $\beta \mathrm{CMa}$ and $\epsilon \mathrm{CMa}$ (Dupin \& Gry 1998; Gry \& Jenkins 2001). We also derive an upper limit of $\log N(\mathrm{Si} \mathrm{IV}) \leqslant 11.25 \mathrm{~cm}^{-2}$ for the HD 158427 sight line, such that the observed ratio of $N(\mathrm{C}$ IV $) / N(\mathrm{Si}$ IV $)>8.9$ is consistent with the predictions of cloud evaporation photoionization models (Slavin \& Frisch 2002).

The $V_{2}=-41.3 \mathrm{~km} \mathrm{~s}^{-1}$ component observed in both of the $\mathrm{C}$ IV lines is not seen in any of the lower ionization UV species. Interstellar gas with such a relatively high velocity is rare within the LC and its presence could be explained if it was associated with an outflow from the nearby Sco-Cen/Loop I superbubble 

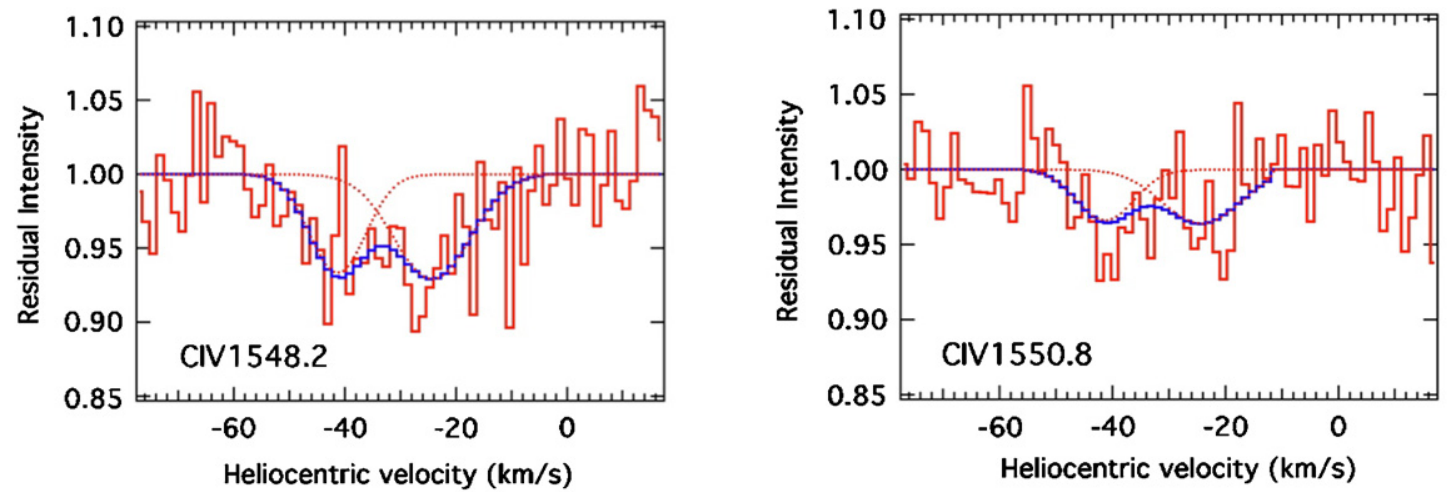

\begin{tabular}{|c|c|c|c|c|c|}
\hline \multicolumn{6}{|c|}{ CIV Line Best Fit Parameters } \\
\hline $\begin{array}{c}\mathbf{V}_{2} \\
(\mathrm{~km} / \mathrm{s}) \\
-41.3+15\end{array}$ & $\begin{array}{c}\mathbf{b}_{2} \\
(\mathrm{~km} / \mathrm{s}) \\
6.8+1.8\end{array}$ & $\begin{array}{c}\log \mathrm{N}_{2} \\
\left(\mathrm{~cm}^{-2}\right) \\
12.04+0.19\end{array}$ & $\begin{array}{c}\mathrm{V}_{1} \\
(\mathrm{~km} / \mathrm{s})\end{array}$ & $\begin{array}{c}\mathbf{b}_{1} \\
(\mathrm{~km} / \mathrm{s}) \\
\mathbf{5}+\mathbf{1}\end{array}$ & $\begin{array}{l}\log \mathbf{N}_{1} \\
\left(\mathrm{~cm}^{-2}\right)\end{array}$ \\
\hline
\end{tabular}
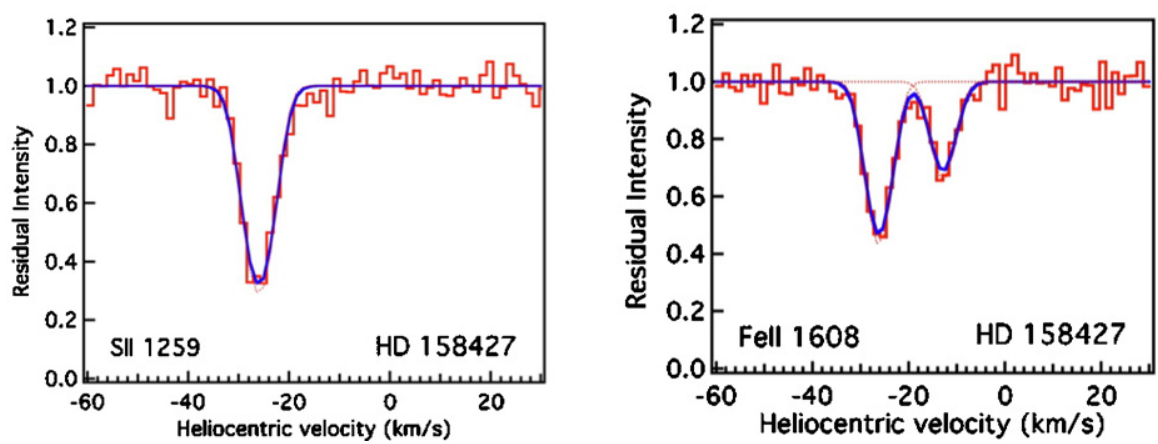

Figure 1. Interstellar absorption profiles of the CIV ( $\lambda 1548$ and $\lambda 1550 \AA)$ lines observed toward HD 158427. Full lines are the model fits superposed upon the normalized residual intensity data points. Dotted lines are the unconvolved components. The best-fit parameters for the two-cloud component fit for both $\mathrm{C}$ IV lines (which were fit simultaneously) are listed in the box. Also shown are the low ionization interstellar lines of S II $1259 \AA$ and Fe II $1608 \AA$ recorded toward this star.

(A color version of this figure is available in the online journal.)

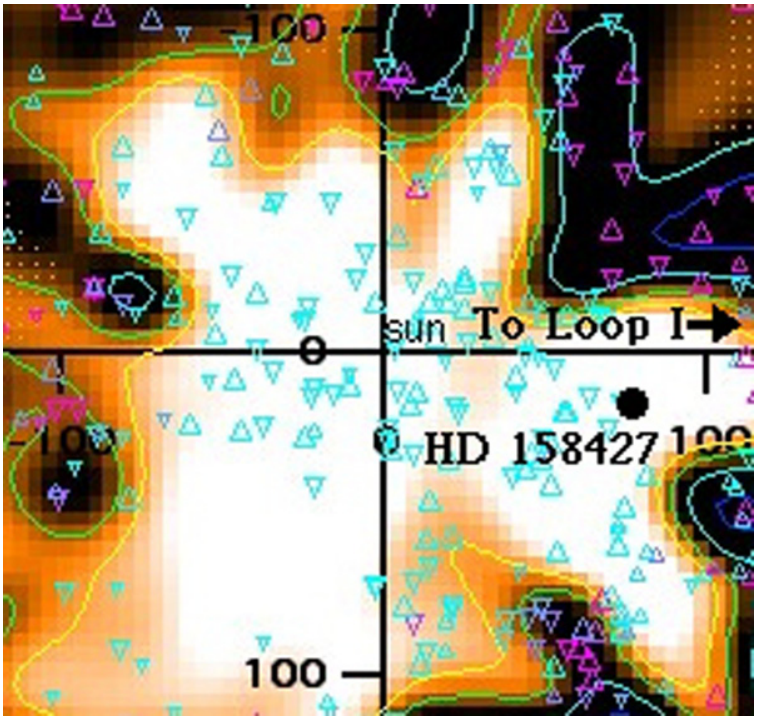

Figure 2. Position of the HD 158427 sight line with respect to the threedimensional spatial contours of the neutral Na I gas within $\pm 100 \mathrm{pc}$ of the Sun as viewed in the galactic plane (Welsh et al. 2010). Dark shading represents dense and cold absorbing gas, whereas light shading represents regions of minimal neutral gas density. Small triangles represent stellar targets whose absorption data were used to construct the map.

(A color version of this figure is available in the online journal.) $\left(l=330^{\circ}, b=+18^{\circ}\right)$. This X-ray emitting interstellar cavity $(d \sim 130 \mathrm{pc})$ is thought to be produced by the collective stellar winds of the Sco-Cen OB association and several consecutive supernova events, such that this bubble is expanding toward the LC with (hot) gas presumably flowing into the LC through the fragmented gap in the boundary wall shown in Figure 2. Support for this view comes from soft X-ray images that show an interaction region between the two interstellar cavities (Egger 1998) and the fact that highly ionized gas with a velocity of $-34 \mathrm{~km} \mathrm{~s}^{-1}$ has been detected flowing away from Loop I by Welsh \& Lallement (2005).

\subsection{Ramifications of the CIV Detection}

High ions produced at an evaporating conductive interface between hot and cooler interstellar gas should possess Doppler line widths that correspond to a thermal gas temperature of $\sim 10^{5} \mathrm{~K}$ (i.e., $b>10 \mathrm{~km} \mathrm{~s}^{-1}$ ). We derive a gas temperature of $\sim 65,000 \mathrm{~K}\left(b=9.5 \mathrm{~km} \mathrm{~s}^{-1}\right)$ for the $V_{1}$ component, which is consistent with what one might expect for the C IV ion. However, the Doppler line width of the $V_{2}$ component is indicative of an unusually low gas temperature of $\leqslant 34,000 \mathrm{~K}$. Narrow Doppler widths can occur for highly ionized gas that is cooling rapidly and out of equilibrium or if it is associated with weak shocks. Similarly, low gas temperatures have been obtained for the Si IV and C IV lines seen by Welsh \& Lallement (2005) toward the Loop I superbubble. 
Although we have detected highly ionized gas associated with the LIC, we note that many other UV observations toward nearby stars have failed to do so, which in many cases may well be due to instrumental sensitivity limitations. The local distribution of the OVI ion has been described as "patchy" (Savage \& Lehner 2006), and this may also apply to the spatial distribution of local C IV (and $\mathrm{Nv}$ ) ions. Although detailing specific theoretical scenarios that might explain this observed "patchiness" is beyond the present scope of this Letter, we offer some speculative thoughts on this point. For example, if $\mathrm{C}_{\mathrm{IV}}$ is generated within turbulent mixing layers (which will depend on an existing shear flow), then a non-uniform flow pattern in the surrounding hot gas could pull off some cold/ warm material from the LIC which then mixes and cools and produces localized CIV gas. Alternately, the geometry of the LIC could be such that it only receives ionizing (soft $\mathrm{X}$-ray) photons along specific sight lines, dependent on the three-dimensional distribution of the intervening opacity of the local ISM. Finally, one can always invoke a peculiar magnetic field topology to control the gas evaporation through thermal conduction.

\section{CONCLUSION}

We have presented high resolution UV absorption measurements of the highly ionized C IV doublet at $\lambda 1550 \AA$ using the refurbished HST STIS instrument. Both line profiles require a two-component cloud structure to fit these data, with one cloud component $\left(V_{1}\right)$ arising at the projected velocity of LIC $\left(-24.3 \mathrm{~km} \mathrm{~s}^{-1}\right)$ and the other $\left(V_{2}\right)$ at $-41.3 \mathrm{~km} \mathrm{~s}^{-1}$. Component $V_{1}$ can almost certainly be associated with absorption arising within a layer of $65,000 \mathrm{~K}$ gas located within $5 \mathrm{pc}$ of the Sun (in the LIC), and its observed C IV column density agrees well with theoretical predictions for the production of this ion in the LIC. However, the Doppler width required to fit the $V_{2}$ gas cloud, which we believe may be associated with an outflow of hot and highly ionized gas from the nearby Loop I superbubble, suggests a low temperature of $\sim 34,000 \mathrm{~K}$ for the C IV gas. These data are part of far larger program of UV absorption observations of local interstellar gas currently being carried out under the Cosmic Origins Spectrograph Science team's guaranteed observer programs using both the HST-STIS and HST-COS instruments during HST Cycles 17 and 18. Therefore, as we receive more of these observations, we believe that they may provide answers to some of the outstanding problems raised in this Letter, partic- ularly with respect to the apparent "patchy" spatial distribution of highly ionized gas absorption within $100 \mathrm{pc}$.

We particularly acknowledge all of the dedicated team of engineers, technicians, scientists, and astronauts who contributed to the success of the STS-125 servicing mission to the Hubble Space Telescope. We thank Jonathan Slavin and John Vallerga for very useful discussions, and also thank the HST-COS science team for their advice and financial support through NASA GSFC grant 005118.

\section{REFERENCES}

Bannister, N., Holberg, J., \& Bruhweiler, F. 2003, MNRAS, 341, 477

Bohringer, H., \& Hartquist, T. 1987, MNRAS, 228, 915

Bruhweiler, F., \& Cheng, K-P. 1988, ApJ, 335, 188

Cowie, L., Taylor, W., \& York, D. 1981, ApJ, 248, 528

Cox, D., \& Helenius, L. 2003, ApJ, 583, 205

Dupin, O., \& Gry, C. 1998, A\&A, 335, 661

Egger, R. 1998, in IAU Colloq. 166, Lecture Notes in Physics, 506, The Local Bubble and Beyond, ed. D. Breitschwerdt, M. Freyberg, \& J. Trumper (Berlin: Springer), 287

Gry, C., \& Jenkins, E. 2001, A\&A, 367, 617

Haffner, L., et al. 2003, ApJS, 149, 405

Hebrard, G., et al. 1999, A\&A, 350, 643

Huang, J-S., Songaila, A., \& Cowie, L. 1995, ApJ, 450, 163

Hurwitz, M., Sasseen, T., \& Sirk, M. 2005, ApJ, 623, 911

Indebetouw, R., \& Shull, J. M. 2004, ApJ, 605, 205

Jelinsky, P., Vallerga, J., \& Edelstein, J. 1995, ApJ, 442, 653

Jenkins, E. 1978, ApJ, 219, 845

Jenkins, E., et al. 2000, ApJ, 538, L81

Koutroumpa, D., Lallement, R., Raymond, J., \& Kharchenko, V. 2009, ApJ, 696, 1517

Lallement, R., Vidal-Madjar, A., \& Ferlet, R. 1986, A\&A, 168, 225

Lallement, R., et al. 1995, A\&A, 304, 461

Lehner, N., et al. 2003, ApJ, 595, 858

Lyu, C-H., \& Bruhweiler, F. 1996, ApJ, 459, 216

Redfield, S. 2009, Space Sci. Rev., 143, 323

Redfield, S., \& Linsky, J. 2004, ApJ, 602, 776

Redfield, S., \& Linsky, J. 2008, ApJ, 673, 283

Robertson, I., \& Cravens, T. 2003, J. Geophys. Res., 108, 6

Savage, B., \& Lehner, N. 2006, ApJS, 162, 134

Slavin, J., \& Cox, D. 1992, ApJ, 392, 131

Slavin, J., \& Frisch, P. 2002, ApJ, 565, 364

Snowden, S., Egger, R., Finkbeiner, D., Freyberg, M., \& Plucinsky, P. 1998, ApJ, 493, 715

Welsh, B. Y., \& Lallement, R. 2005, A\&A, 436, 615

Welsh, B. Y., Lallement, R., Vergely, J-L., \& Raimond, S. 2010, A\&A, 510, 54

Welsh, B. Y., \& Shelton, R. 2009, Astrophys. Space Sci., 323, 1

Wolff, B., Koester, D., \& Lallenment, R. 1999, A\&A, 346, 969

Wood, B., Redfield, S., Linsky, J., \& Sahu, M. 2002, ApJ, 581, 1168

Woodgate, B., et al. 1998, PASP, 110, 1183 